\title{
Erratum to: Increased risk of tumor in DM1 is not related to exposure to common lifestyle risk factors
}

\author{
Maria Laura Ester Bianchi ${ }^{1}$ - Emanuele Leoncini ${ }^{2}$ - Marcella Masciullo $^{3}$. \\ Anna Modoni ${ }^{1}$ - Shahinaz M. Gadalla ${ }^{4} \cdot$ Roberto Massa $^{5}$ - Annalisa Botta ${ }^{10}$. \\ Emanuele Rastelli $^{5}$ - Chiara Terracciano ${ }^{5}$ - Giovanni Antonini ${ }^{6} \cdot$ Elisabetta Bucci $^{6}$. \\ Antonio Petrucci $^{7} \cdot$ Sandro Costanzi $^{8} \cdot$ Massimo Santoro $^{9} \cdot$ Stefania Boccia $^{2}$. \\ Gabriella Silvestri ${ }^{1}$
}

Published online: 25 February 2016

(C) Springer-Verlag Berlin Heidelberg 2016

\section{Erratum to: J Neurol \\ DOI 10.1007/s00415-015-8006-y}

Unfortunately, one co-author's name was not listed in the original publication. The missing author name and affiliation is given below:

Annalisa Botta

Department of Biomedicine and Prevention, Tor Vergata University, Rome, Italy

The online version of the original article can be found under doi:10.1007/s00415-015-8006-y.

Gabriella Silvestri

gsilvestri@ rm.unicatt.it

1 Department of Geriatrics, Neuroscience and Orthopedics, Università Cattolica del Sacro Cuore, Largo F. Vito 1, 00168 Rome, Italy

2 Institute of Public Health, Section of Hygiene, UCSC, Rome, Italy

3 IRCSS Fondazione Santa Lucia, Rome, Italy

4 Clinical Genetics Branch, Division of Cancer Epidemiology and Genetics, National Cancer Institute, Bethesda, MD, USA

5 Department of Systems Medicine (Neurology), University of Rome Tor Vergata, Rome, Italy
6 Department of Neurosciences, Mental Health and Sensory Organs (NESMOS), Sapienza University of Rome, Rome, Italy

7 Unità Operativa Complessa Neurologia e Neurofisiopatologia, Azienda Ospedaliera San Camillo Forlanini, Rome, Italy

8 Unit of Medical Genetics, Center for Neuromuscular and Neurological Rare Diseases, S. Camillo Forlanini Hospital, Rome, Italy

9 Fondazione Don Carlo Gnocchi Onlus, Milan, Italy

10 Department of Biomedicine and Prevention, Tor Vergata University, Rome, Italy 